\title{
Optical shaping of plasma cavity for controlled laser wakefield acceleration
}

\author{
Bobbili Sanyasi Rao $\odot,{ }^{1,2, *}$ Myung Hoon Cho $\odot,{ }^{1,3}$ Hyung Taek Kim $\odot,{ }^{1,4, \dagger}$ Jung Hun Shin, ${ }^{1}$ Kyung Hwan Oh, ${ }^{1}$ \\ Jong Ho Jeon, ${ }^{1}$ Byung Ju Yoo, ${ }^{1}$ Seong Ha Cho, ${ }^{1}$ Jin Woo Yoon, ${ }^{1,4}$ Jae Hee Sung, ${ }^{1,4}$ Seong Ku Lee, ${ }^{1,4}$ and Chang Hee Nam $\odot{ }^{1,5}$ \\ ${ }^{1}$ Center for Relativistic Laser Science (CoReLS), IBS, Gwangju 61005, Korea \\ ${ }^{2}$ Laser Plasma Division, Raja Ramanna Centre for Advanced Technology, HBNI, Indore 452013, India \\ ${ }^{3}$ Pohang Accelerator Laboratory, Pohang, Gyeongbuk 37673, Korea \\ ${ }^{4}$ Advanced Photonics Research Institute, GIST, Gwangju 61005, Korea \\ ${ }^{5}$ Department of Physics and Photon Science, GIST, Gwangju 61005, Korea
}

(Received 22 May 2020; revised 21 September 2020; accepted 4 November 2020; published 4 December 2020)

\begin{abstract}
Laser wakefield accelerators rely on relativistically moving micrometer-sized plasma cavities that provide extremely high electric field $>100 \mathrm{GV} / \mathrm{m}$. Here, we demonstrate transverse shaping of the plasma cavity to produce controlled sub-GeV electron beams, adopting laser pulses with an axially rotatable ellipse-shaped focal spot. We showed the control capability on electron self-injection, charge, and transverse profile of the electron beam by rotating the focal spot. We observed that the effect of the elliptical focal spot was imprinted in the profiles of the electron beams and the electron energy increased, as compared to the case of a circular focal spot. We performed three-dimensional particle-in-cell simulations which reproduced the experimental results and revealed dynamics of an asymmetric self-injection process. This simple scheme offers a control method on laser wakefield acceleration to produce tailored electron beams and $\mathrm{x}$ rays for various applications.
\end{abstract}

DOI: 10.1103/PhysRevResearch.2.043319

\section{INTRODUCTION}

The laser wakefield acceleration (LWFA) has attracted attention intensively since its conception [1-3] as a potential alternative to the conventional technology for developing a compact $\mathrm{TeV}$ electron-positron collider [4] and ultrashort bright X-ray sources [5]. Recently, nearly $10-\mathrm{GeV}$ electron beams have been demonstrated through LWFA from 10$\mathrm{cm}$ scale plasma with a petawatt laser pulse [6,7]. Further, significant efforts are devoted to the efficient control of the injection and acceleration processes to produce controllable high-quality electron beams [8-16] and bright radiation sources [17-21] for a variety of applications.

LWFA exploits strong wakefields $(\geqslant 100 \mathrm{GV} / \mathrm{m})$ in plasma excited by the ponderomotive force $\left(F_{p}\right)$ of an intense laser pulse. The ponderomotive force $\left(F_{p} \propto-\nabla I_{L}\right.$, where $I_{L}$ is laser intensity) of a focused short-pulse laser displaces plasma electrons in all directions, while heavier ions stay behind. The charge separation forms a spherical plasma wave, so-called plasma cavity or bubble, when the pulse length and focal spot radius of the laser match half the plasma wavelength [22,23]. At sufficiently high intensity the laser pulse induces nonlinear plasma wave breaking which causes self-injection of elec-

\footnotetext{
*sunnyb@ rrcat.gov.in

†htkim@gist.ac.kr

Published by the American Physical Society under the terms of the Creative Commons Attribution 4.0 International license. Further distribution of this work must maintain attribution to the author(s) and the published article's title, journal citation, and DOI.
}

tron bunch into the plasma cavity from its back end [24]. The injected electrons experience accelerating and focusing forces due to axial and transverse components of the wakefield in the plasma cavity, respectively. The radial force acts to transversely confine the accelerating electrons and causes their transverse oscillations, known as "betatron oscillations" around the laser axis, which produces highly directional ultrashort $(\sim \mathrm{fs})$ synchrotron radiation [21].

When a laser pulse is focused to a perfect circular spot, the associated ponderomotive force produces a transversely symmetric plasma cavity and wakefield. Studies on electron injection in LWFA [25,26] and emission of betatron radiation [19] mostly consider spherical plasma cavities. In principle, shaped laser focal spot can tailor the transverse shape of the plasma cavity for delicate control of electrons' self-injection and their trajectories, which can then facilitate generation of controlled electron beams and radiation. Earlier, the effects of off-centered intensity profile or an astigmatic aberration of drive laser pulse have been studied to manipulate betatron oscillations and radiation [20,27-29]. However, direct control of the LWFA and transverse properties of the electron beam by shaping the laser focus have not been shown so far, even though the tuning of LWFA by manipulating the plasma cavity can play a crucial role in better understanding and future development of LWFA.

We report here demonstration of stable sub-GeV quasimonoenergetic electron beams with enhanced energy and tailored transverse profile from LWFA driven by a laser pulse with an ellipse-shaped focal spot. We show that the orientation, ellipticity, and charge of the transversely ellipse-shaped electron beam could be tuned by simply rotating the focal spot. We proved in experiments and particle-in-cell (PIC) simula- 


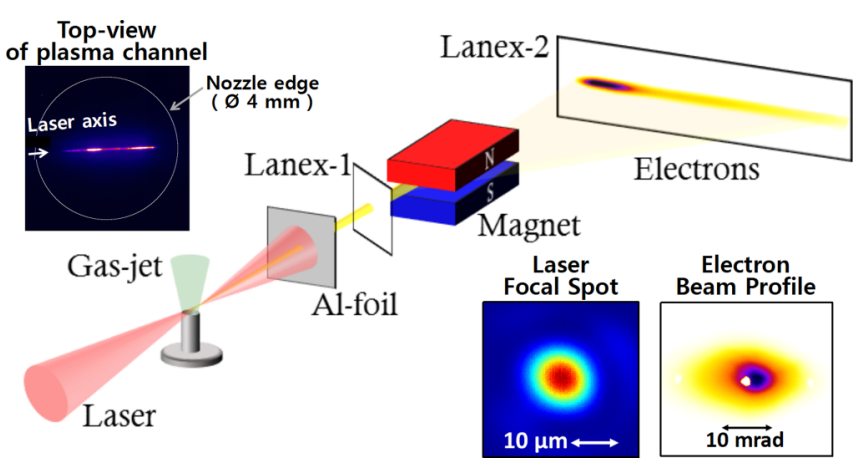

FIG. 1. Schematic of the experimental setup used for laser wakefield acceleration. The images of the laser focal spot and the corresponding electron-beam profile without shaping of the laser beam are shown in the inset at bottom right. The top-view image of the plasma channel is shown in the inset at top left. The focal spot was nearly circular after correcting with an adaptive optics.

tions that the shaped laser focus could be effectively used for shaping the plasma cavity and fine control of self-injection in LWFA, which can be exploited for producing tunable electron and $\mathrm{x}$-ray beams in the future.

\section{EXPERIMENTAL SETUP}

The experiment was performed using a femtosecond Ti:sapphire laser with a peak power of $150 \mathrm{TW}$. The laser delivered horizontally polarized ultrashort laser pulses of full width at half maximum (FWHM) duration, $\tau_{\mathrm{L}}=27 \pm 2 \mathrm{fs}$. The transverse profile of the laser beam before focusing was circular and had a flat-top intensity distribution with measured FWHM diameter of $70 \mathrm{~mm}$. A schematic of the experimental setup is shown in Fig. 1. The laser beam was focused, using a spherical focusing mirror with f-number 17 , into a helium gas jet produced from a cylindrical nozzle with an orifice diameter of $4 \mathrm{~mm}$. The density profile of the gas jet was measured using interferometry. The laser focus was kept at a height of $1 \mathrm{~mm}$ from the nozzle where the gas jet has 2-mm-wide flat-top density profile with 1-mm up- and down ramps. The laser propagation and its interaction length in the helium gas jet plasma were monitored through top-view imaging of the plasma radiation.

The electron beams produced by the LWFA were characterized using the diagnostics setup in the laser downstream direction. A thin aluminum foil (Al foil), kept after the interaction, blocks the residual drive laser beam. The scintillating screens (Lanex ${ }^{\mathrm{TM}}$ ) Lanex-1 (kept at a distance of $35 \mathrm{~cm}$ from the gas jet) and Lanex-2 (kept at a distance of $52 \mathrm{~cm}$ from the exit of the magnet) were covered on front with $0.1-\mathrm{mm}$ thick Al foils to cut off low-energy background electrons, and rear sensitive surfaces are imaged with charge-coupled device cameras for monitoring electron beam before and after the magnet. The images obtained from the Lanex-1 were used to measure the pointing, transverse shape, divergence, and total charge of the electron beam. A permanent dipole magnet, having $0.96 \mathrm{~T}$ over $20.5-\mathrm{cm}$ length within a pole gap of 8 $\mathrm{mm}$, was used to measure the electron-energy spectrum. The Lanex-2 screen was set to detect electrons in the energy range $0.1-0.5 \mathrm{GeV}$. The resolution of electron-energy measurement

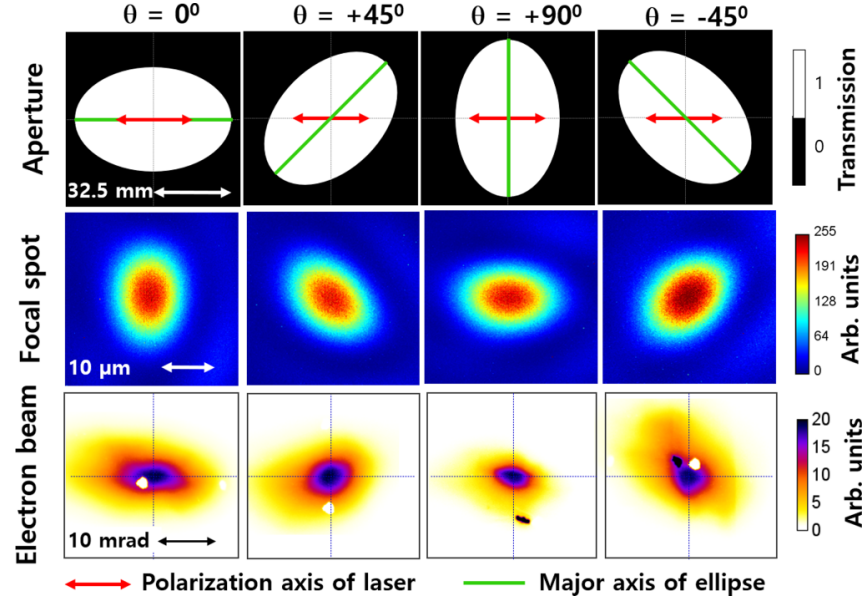

FIG. 2. Top row: representative images of the ellipse-shaped hard aperture oriented in four different directions with respect to the laser polarization direction, and the corresponding images of the laser focal spot and the typical electron-beam profile recorded in a single shot when the aperture was introduced in the drive laser beam. The white dots in the electron-beam images are reference markers on Lanex screen used for the calibration of imaging.

was $2 \%$ at $0.1 \mathrm{GeV}$ and $16 \%$ at $0.5 \mathrm{GeV}$ by considering the beam divergence of $10 \mathrm{mrad}$.

We have employed an adaptive optics system to correct the wavefront distortion of the laser beam to avoid the effects of wave-front aberration and obtain a high-quality focal spot. A typical image of measured focal spot is shown in an inset of Fig. 1. The focal spot was nearly circular with average $w_{0}$ of $17 \mu \mathrm{m}$. For the focal-spot shaping, we introduced an ellipseshaped hard aperture in the laser beam path centered on the beam axis, prior to sending the laser beam onto the focusing mirror. The elliptic aperture has a diameter of $65 \mathrm{~mm} \times 43.3$ $\mathrm{mm}$ and ellipticity of 1.5 . The aperture could be translated in and out of the beam path and could also be rotated about the axis of the laser beam. The introduction of the aperture caused the laser beam to acquire ellipse shape and transmitted nearly $60 \%$ of the laser energy.

The focal-spot images of the ellipse-shaped laser beam were measured for different orientations of the ellipse with respect to the laser polarization axis, as shown in Fig. 2. The focal spot was clearly elliptical with its major axis aligned perpendicular to that of the elliptical aperture. The rotation of the focal spot with the rotation of aperture could also be noticed in the figure. The $1 / e^{2}$ radius of the shaped focal spot was measured to be $25 \mu \mathrm{m} \times 17 \mu \mathrm{m}$, which gives rise to the ellipticity, $e \approx 1.5$, where we define ellipticity $e=a / b$; $a$ and $b$ are, respectively, the major and the minor radius of the ellipse. The peak laser intensity $I_{L}$ in the focus is $8.4 \times 10^{18} \mathrm{~W} / \mathrm{cm}^{2}$ and the corresponding normalized vector potential, $a_{0}=0.85\left[I_{L}\left(\times 10^{18} \mathrm{~W} / \mathrm{cm}^{2}\right) \lambda^{2}(\mu \mathrm{m})\right]^{1 / 2} \approx 2$, considering the transmission of the aperture and the energy concentrated in the focal spot. Corresponding to the $60 \%$ transmission of the aperture, the laser beam without aperture provided higher peak intensity of $1.7 \times 10^{19} \mathrm{~W} / \mathrm{cm}^{2}$ and $a_{0} \approx 2.5$. 

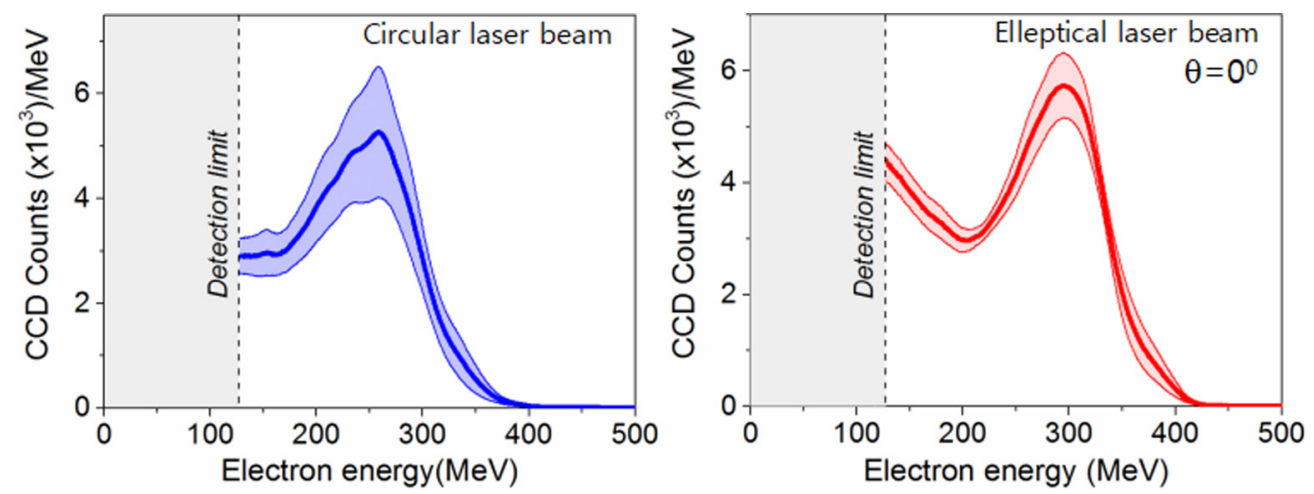

FIG. 3. Experimentally measured energy spectra of electron beams produced from LWFA with circular and elliptical focus.

\section{EXPERIMENTAL RESULTS}

The LWFA experiment was initially carried out without the elliptic aperture and reproducible electron beams were obtained from self-injected LWFA at plasma density, $n_{e} \approx$ $1 \times 10^{19} \mathrm{~cm}^{-3}\left(c \tau=0.8 \lambda_{p}>\lambda_{p} / 2\right)$ from $2.3 \pm 0.1$-mm-long plasma channel. The laser power was reduced to about $60 \%$ of maximum power in order to match the laser energy on target with and without aperture. The electron-beam measurements were performed in 20-30 laser shots for each set of experimental conditions and obtained the mean and the standard error [30] of the mean for the relevant beam parameters. The standard error was determined from the standard deviation of the individual shots divided by the square root of the number of shots in each set. Images of the typical electron-beam profile and the laser-plasma channel are shown in the insets of Fig. 1. In spite of the circular focal spot, the electron-beam profile elongated along the laser polarization axis, indicating the overlap of accelerating electrons with the drive laser field inside the back half of the bubble [31]. The direct influence of the laser field was further evident during the experiment from the observation of larger ellipticity at higher plasma density and/or laser power. The FWHM contour of the electron-beam profile was fitted to a closest ellipse to quantify the ellipticity of the electron beam. The electron beam has divergence angles $10.2 \pm 0.7$ and $6.0 \pm 0.4 \mathrm{mrad}$ along the horizontal and the vertical planes, respectively. The ellipticity of the electron beam was $1.74 \pm 0.10$ and the orientation of its major axis was $-5.2^{\circ} \pm 3.8^{\circ}$. The rms pointing fluctuations of the electron beams were $1.9 \mathrm{mrad}$ and $1.7 \mathrm{mrad}$ in both the planes.

To demonstrate the effect of ellipse-shaped focal spot on LWFA, the elliptical aperture was inserted in the laser beam. The images in the third row of Fig. 2 show the typical single-shot profiles of electron beams recorded for different orientations of the focal spot with respect to the laser polarization axis. Figure 2 clearly indicates that the major axis of the electron beam follows the rotation angle of the aperture except for the case of $\theta=90^{\circ}$. In the case of $\theta=90^{\circ}$, the major axis of the elliptic electron beam possibly could not be aligned with that of the aperture due to the strong effect of the laser polarization along the horizontal axis (see Appendix).

The energy spectra of the electron beams were compared for the two cases obtained with and without the aperture in the laser-beam path for the same laser energy on target. We found that the shaped laser focus can stabilize the electron injection resulting in electron beams with better reproducibility. The averaged energy spectra with rms errors measured with and without the aperture are shown in Fig. 3. The electron beams with the elliptic focal spot had enhanced stability and higher peak energy compared to the circular focus result. We could not observe considerable change in the peak energy of the electron beam with the rotation of the focal spot.

The effect of the shaped focal spot on the spatial parameters and the charge of the electron beam is shown in detail in Fig. 4. As discussed earlier, the major axis of an electron beam obviously rotated with the rotation of the aperture except for the orientation of $90^{\circ}$. However, the effect of asymmetric wakefield on the transverse profile of the electron beam can be identified from Fig. 4(b). The angular divergence along the major axis is highest for the $0^{\circ}$ orientation of the aperture and the lowest for $90^{\circ}$, which highlights the asymmetry (higher strength along the minor axis of the focus) of transverse forces. The variation of different spatial properties of the electron beam shown in Fig. 4 originates from the combined effect of the drive laser field and the asymmetry in the transverse wakefield caused by the elliptical focal spot. These results clarify the crucial role of transverse forces of the wakefield in controlling LWFA and electron trajectories. More interestingly, the charge of the accelerated electron beam shows a strong dependence on the orientation of the elliptical focal spot with respect to the polarization axis. This indicates the delicate influence of the transverse asymmetry of wakefield on electron injection dynamics. It should be noted that the highest charge is injected when the major axis of the elliptical spot is perpendicular to the laser polarization axis and the least charge (33\% of maximum charge) is injected for $\pm 45^{\circ}$. The difference in the charge for $0^{\circ}$ and $90^{\circ}$ is also noticeable, although the difference of about $25 \%$ is less drastic compared to the cases of $\pm 45^{\circ}$.

\section{PARTICLE-IN-CELL SIMULATIONS}

We performed three-dimensional (3D) PIC simulation using the code JOPIC [32] for plasma density of $4.5 \times 10^{18} \mathrm{~cm}^{-3}$ and for the horizontally polarized laser pulses with duration, $\tau=25 \mathrm{fs}$ ( $\approx$ half of the plasma wave period) and circular (elliptical) focal-spot size $20 \mu \mathrm{m}(30 \mu \mathrm{m} \times 20 \mu \mathrm{m})$ at FWHM. The energy content for both cases is kept constant, i.e., $a_{0}=$ 2.7 for the circular focus and 2.2 for the elliptical focus. The 

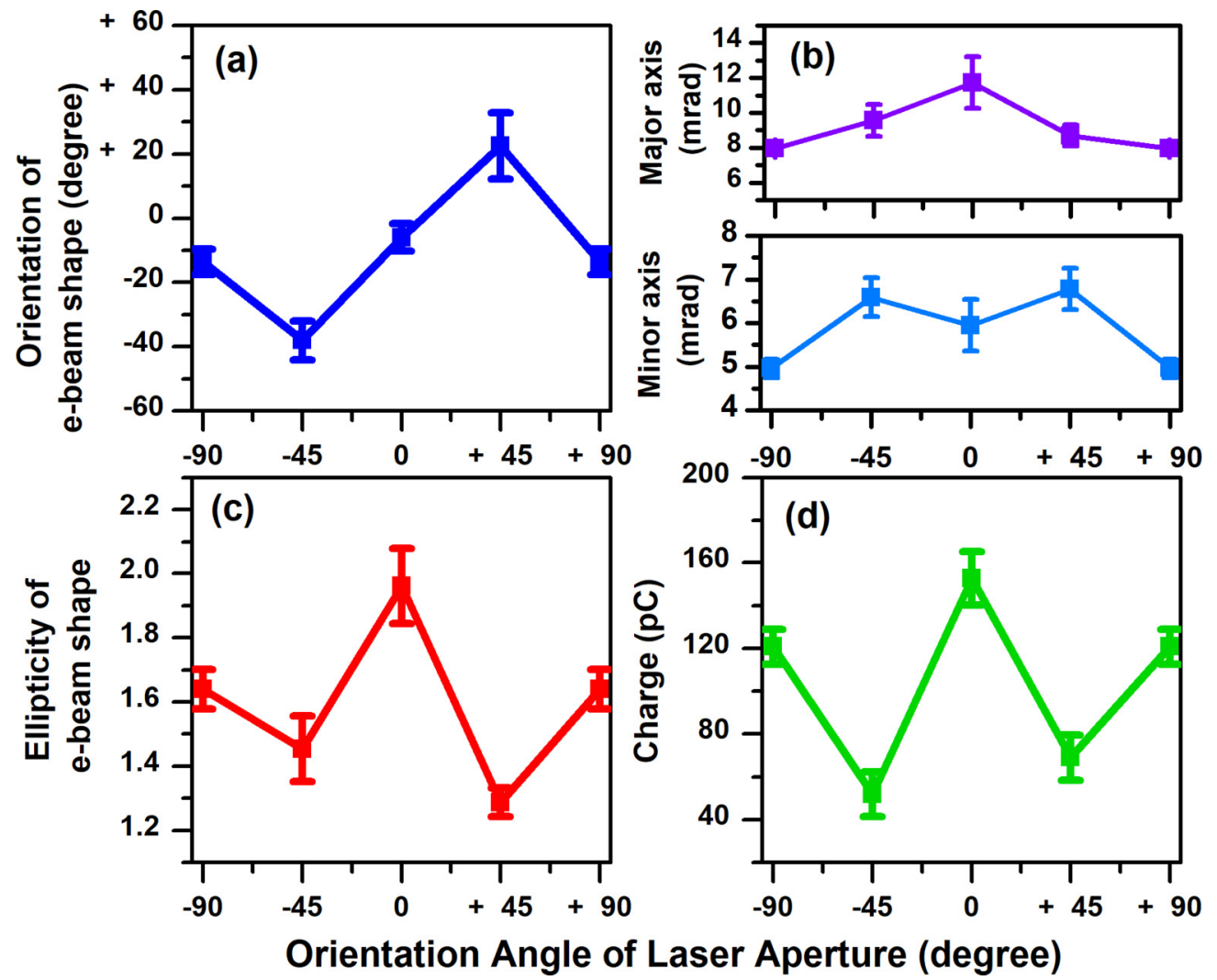

FIG. 4. Variation of electron-beam parameters with change in orientation of the ellipse-shaped laser beam with respect to its polarization. For comparison, the electron-beam parameters with circular laser beam are (a) orientation: $-5.2^{\circ} \pm 3.8^{\circ}$, (b) horizontal divergence: $10.2 \pm$ $0.73 \mathrm{mrad}$ and vertical divergence: $6.0 \pm 0.44 \mathrm{mrad}$, (c) ellipticity: $1.7 \pm 0.10$, and (d) charge: $120 \pm 10 \mathrm{pC}$.

plasma density of $4.5 \times 10^{18} \mathrm{~cm}^{-3}$ was chosen to avoid the direct influence of the laser field on accelerating electrons and clearly investigate the effect of shaped cavity alone on the dynamic of LWFA. The plasma density profile is set close to the experimental conditions and the laser focal position is $3 \mathrm{~mm}$ where it is behind the plasma target from the middle of the gas jet. Simulations clearly show an electron beam with elliptic profile [Fig. 5(a)] and higher energy [Fig. 5(b)], even though the initial $a_{0}$ of the elliptical laser focus is lower than the circular focal spot, as observed in the experiment.

Figure 5(c) shows the snapshots at the moment of selfinjection in the plasma cavity. The circular focal spot produced transversely circular-shaped plasma cavity and the electron injection occurred symmetrically, as expected. The formation of transversely elliptic (ellipsoid in 3D)-shaped plasma cavity can be clearly seen for the elliptic focal spot. It is interesting to note that the electron injection occurs asymmetrically with the elliptical focal spot wherein more electrons are injected in the vertical plane following the major axis of the laser focus although the transverse wakefield along the major axis is weaker compared to that along the minor axis. The injected electrons acquire larger transverse momentum along the minor axis due to stronger transverse wakefield and produce horizontally ellipse-shaped electron beam as shown in Fig. 5(a). The results clearly show that the elliptic-shaped laser can be effectively used to control the trajectories of plasma electrons and confine the self-injection in a desired plane which is otherwise not possible in conventional LWFA.

The simulations also show enhanced electron energy with elliptic laser focus [see Fig. 5(b)] which can be explained by considering the injection moment. Figure $5(\mathrm{~d})$ shows the evolution of the axial wakefield with its propagation distance in the plasma. It shows that sufficient wakefield strength for self-injection develops earlier [dashed line in Fig. 5(d)] in the case of elliptical focal spot. The earlier injection, therefore, facilitates extended acceleration and higher-energy electron beam as shown in Fig. 5(b). Both cases indicate that the injection occurs at about $a_{0}=3$. Even though the elliptical focal spot has lower initial $a_{0}$, the broader spot size along the major axis produces higher $a_{0}$ earlier and drives stronger wakefield than that of the circular spot.

\section{DISCUSSION}

In previous studies, LWFA with asymmetric plasma cavity has been studied with a tilted wave front [28] or astigmatic aberration [29] of a driving laser pulse to control electronbeam direction or to study the angular-momentum evolution of electron beams, respectively. In addition, the generation of an elliptic electron beam along the laser polarization axis, as we observed with circular laser spot, was studied to originate from the direct laser interaction of an accelerated electron beam in the first cavity [31]. In this study, we proved that the elliptically shaped laser beams without 

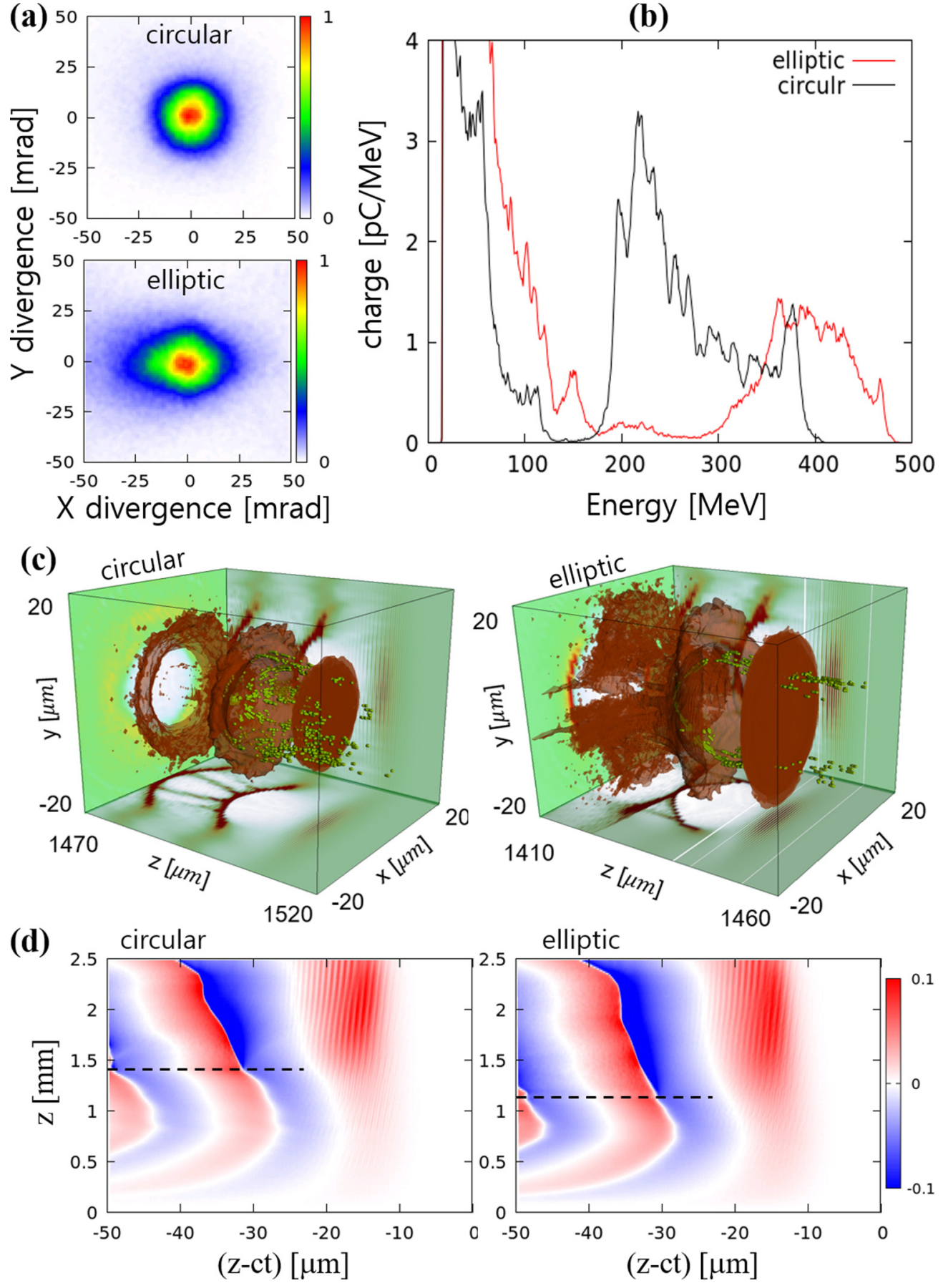

(b)

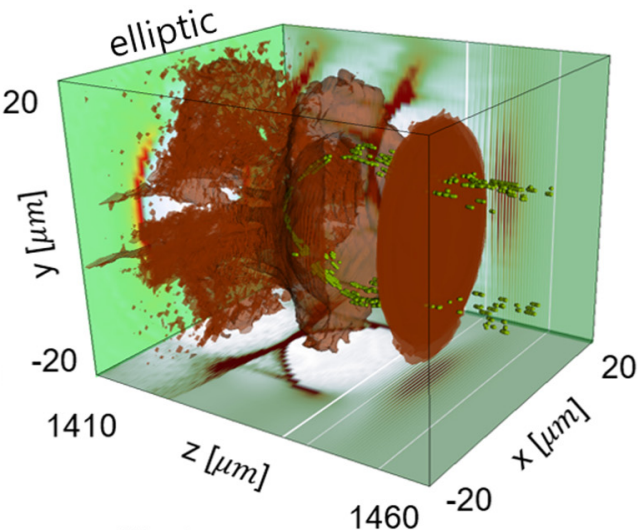
(1)

FIG. 5. 3D PIC simulation results of LWFA with circular and elliptical focal spots containing the same laser energy: (a) spatial profiles, (b) energy spectra of the electron beams, (c) electron injection in the plasma cavity, and (d) evolution of the wakefield (injection point shown with a dashed horizontal line).

aberrations or wave-front tilts can be an intrinsic and direct method to control the plasma cavity structure that results in the imprinting of laser profile on the electron beam. The apparent correlation between laser focal spot and electron beam could be verified owing to the aberration-free laser beam obtained with the adaptive control of laser wave front. We could also decouple the effect of asymmetric plasma cavity and laser polarization by observing the electron-beam shape rotating with the elliptic focal shape. Our investigations clearly demonstrated that not only the transverse 


\section{CONCLUSIONS}

In conclusion, we demonstrated that the transverse shaping of a laser focal spot improved the stability and energy of electron beams produced by effectively controlling the transverse shape of plasma cavities in LWFA. The cavity shaping and its rotation provided control on electron injection dynamics and the consequent variation of accelerated electron charge, energy, and the plane of betatron oscillations. With adaptive optics available at high-power laser facilities, in situ focal-spot shaping can be routinely done in a feedback loop to accomplish shaped electron and polarized x-ray beams at target location for future research and applications of LWFA.

\section{ACKNOWLEDGMENTS}

This work was supported by the Institute for Basic Science under Grant No. IBS-R012-D1. H.T.K. and S.K.L. were partially supported by GIST Research Institute (GRI) grant funded by the GIST in 2020. H.T.K. was also partly supported by the National Research Foundation of Korea (NRF) grant funded by the Korea government (MSIT) Grant No. NRF2020R1F1A1070538.

\section{APPENDIX}

The ellipticity of the electron beam due to interaction with the horizontally polarized direct laser field can be compen- sated partly by the elliptical focal spot orientation of $\theta=90^{\circ}$, as reflected in the measurements. However, there will be two equal components of the laser electric field act along the major and minor axes of the electron beam for the case $\theta= \pm 45^{\circ}$. This will increase both the major and minor axes of the elliptical electron beam by the same magnitude and causes the resulting ellipticity to decrease. If the elongation of the electron beam by elliptic focal spot is smaller than that by the direct laser interaction, the electron beam can have lesser ellipticity for $\theta= \pm 45^{\circ}$ compared to $\theta= \pm 90^{\circ}$. This can be quantitively understood by formulating the contributions to divergence along each axis individually from the elliptic focal spot and direct laser interaction. If we assume $\theta_{0}$ as the divergence of the circular electron beam by circular focal spot, and $\delta \theta_{L}$ and $\delta \theta_{\mathrm{PC}}$ (where $0<\delta \theta_{L}<\theta_{0}$ and $\left.0<\delta \theta_{\mathrm{PC}}<\theta_{0}\right)$ are the additional components of divergence arising due to the direct laser field and elliptic plasma cavity, respectively, then we can formulate the ellipticity of the electron beam $e=\left(\theta_{0}+\delta \theta_{L}\right) /\left(\theta_{0}+\delta \theta_{\mathrm{PC}}\right)$ for the cases $\theta= \pm 90^{\circ}$ and $e^{\prime}=\left(\theta_{0}+\delta \theta_{\mathrm{PC}}+\delta \theta_{L} \cos 45^{\circ}\right) /\left(\theta_{0}+\delta \theta_{L} \cos 45^{\circ}\right)$ for $\theta=$ $\pm 45^{\circ}$. We shall find that $e^{\prime}<e$ for $\delta \theta_{\mathrm{PC}} / \delta \theta_{L}<0.5$ and the electron beams appear more round for $\theta= \pm 45^{\circ}$ compared to $\theta= \pm 90^{\circ}$. For example, if we take $\theta_{0}=6 \mathrm{mrad}, \delta \theta_{L}=$ $4.5 \mathrm{mrad}$, and $\delta \theta_{\mathrm{PC}}=1.5 \mathrm{mrad}$ close to our experimental conditions, we find that $e \approx 1.4$ for $\theta=90^{\circ}$ and $e^{\prime} \approx 1.2$ for $\theta=45^{\circ}$ which is in good agreement with the observed results.
[1] T. Tajima and J. M. Dawson, Laser Electron Accelerator, Phys. Rev. Lett. 43, 267 (1979).

[2] E. Esarey, C. B. Schroeder, and W. P. Leemans, Physics of laserdriven plasma-based electron accelerators, Rev. Mod. Phys. 81, 1229 (2009).

[3] C. Joshi, Laser-driven plasma accelerators operating in the selfguided, blowout regime, IEEE Trans. Plasma Sci. 45, 3134 (2017).

[4] C. B. Schroeder, E. Esarey, C. G. R. Geddes, C. Benedetti, and W. P. Leemans, Physics considerations for laser-plasma linear colliders, Phys. Rev. ST Accel. Beams 13, 101301 (2010).

[5] M. E. Couprie, A. Loulergue, M. Labat, R. Lehe, and V. Malka, Towards a free electron laser based on laser plasma accelerators, Phys. Rev. ST Accel. Beams 47, 234001 (2014).

[6] H. T. Kim, J. H. Shin, C. Aniculaesei, B. S. Rao, V. B. Pathak, M. H. Cho, C. Hojbota, S. K. Lee, J. H. Sung, H. W. Lee, J. W. Yoon, K. Nakajima, and C. H. Nam, Multi-PW laser driven electron acceleration and applications, The 46th European Physical Society Conference on Plasma Physics (Europian Physical Society, 2019), p. I1.204.

[7] A. J. Gonsalves, K. Nakamura, J. Daniels, C. Benedetti, C. Pieronek, T. C. H. de Raadt, S. Steinke, J. H. Bin, S. S. Bulanov, J. van Tilborg, C. G. R. Geddes, C. B. Schroeder, Cs. Tóth, E. Esarey, K. Swanson, L. Fan-Chiang, G. Bagdasarov, N. Bobrova, V. Gasilov, G. Korn, P. Sasorov, and W. P. Leemans, Petawatt Laser Guiding and Electron Beam Acceleration to 8 $\mathrm{GeV}$ in a Laser-Heated Capillary Discharge Waveguide, Phys. Rev. Lett. 122, 084801 (2019).
[8] M. H. Cho, V. B. Pathak, H. T. Kim, and C. H. Nam, Controlled electron injection facilitated by nanoparticles for laser wakefield acceleration, Sci. Rep. 8, 16924 (2018).

[9] J. Faure, C. Rechatin, A. Norlin, A. Lifschitz, Y. Glinec, and V. Malka, Controlled injection and acceleration of electrons in plasma wakefields by colliding laser pulses, Nature (London) 444, 737 (2006).

[10] A. Pak, K. A. Marsh, S. F. Martins, W. Lu, W. B. Mori, and C. Joshi, Injection and Trapping of Tunnel-Ionized Electrons into Laser-Produced Wakes, Phys. Rev. Lett. 104, 025003 (2010).

[11] B. B. Pollock, C. E. Clayton, J. E. Ralph, F. Albert, A. Davidson, L. Divol, C. Filip, S. H. Glenzer, K. Herpoldt, W. Lu, K. A. Marsh, J. Meinecke, W. B. Mori, A. Pak, T. C. Rensink, J. S. Ross, J. Shaw, G. R. Tynan, C. Joshi, and D. H. Froula, Demonstration of a Narrow Energy Spread, $\sim 0.5 \mathrm{GeV}$ Electron Beam from a Two-Stage Laser Wakefield Accelerator, Phys. Rev. Lett. 107, 045001 (2011).

[12] A. J. Gonsalves, K. Nakamura, C. Lin, D. Panasenko, S. Shiraishi, T. Sokollik, C. Benedetti, C. B. Schroeder, C. G. R. Geddes, J. van Tilborg, J. Osterhoff, E. Esarey, C. Toth, and W. P. Leemans, Tunable laser plasma accelerator based on longitudinal density tailoring, Nat. Phys. 7, 862 (2011).

[13] B. S. Rao, A. Moorti, R. Rathore, J. A. Chakera, P. A. Naik, and P. D. Gupta, High-quality electron beam from laser wake-field acceleration in laser produced plasma plumes, Appl. Phys. Lett. 102, 231108 (2013).

[14] A. Buck, J. Wenz, J. Xu, K. Khrennikov, K. Schmid, M. Heigoldt, J. M. Mikhailova, M. Geissler, B. Shen, F. Krausz, 
S. Karsch, and L. Veisz, Shock-Front Injector for High-Quality Laser-Plasma Acceleration, Phys. Rev. Lett. 110, 185006 (2013).

[15] R. Lehe, A. F. Lifschitz, X. Davoine, C. Thaury, and V. Malka, Optical Transverse Injection in Laser-Plasma Acceleration, Phys. Rev. Lett. 111, 085005 (2013).

[16] B. S. Rao, A. Moorti, R. Rathore, J. A. Chakera, P. A. Naik, and P. D. Gupta, High-quality stable electron beams from laser wakefield acceleration in high density plasma, Phys. Rev. ST Accel. Beams 17, 011301 (2014).

[17] J. Wenz, J. Wenz, A. Döpp, K. Khrennikov, S. Schindler, M. F. Gilljohann, H. Ding, J. Götzfried, A. Buck, J. Xu, M. Heigoldt, W. Helml, L. Veisz, and S. Karsch, Dual-energy electron beams from a compact laser-driven accelerator, Nat. Photonics 13, 263 (2019).

[18] J. Luo, M. Chen, M. Zeng, J. Vieira, L. L. Yu, S. M. Weng, L. O. Silva, D. A. Jaroszynski, Z. M. Sheng, and J. Zhang, A compact tunable polarized X-ray source based on laser-plasma helical undulators, Sci. Rep. 6, 29101 (2016).

[19] S. Corde, K. Ta Phuoc, R. Fitour, J. Faure, A. Tafzi, J. P. Goddet, V. Malka, and A. Rousse, Controlled Betatron X-Ray Radiation from Tunable Optically Injected Electrons, Phys. Rev. Lett. 107, 255003 (2011).

[20] S. P. D. Mangles, G. Genoud, S. Kneip, M. Burza, K. Cassou, B. Cros, N. P. Dover, C. Kamperidis, Z. Najmudin, A. Persson, J. Schreiber, F. Wojda, and C.-G. Wahlström, Controlling the spectrum of x-rays generated in a laser-plasma accelerator by tailoring the laser wavefront, Appl. Phys. Lett. 95, 181106 (2009).

[21] S. Corde, K. Ta Phuoc, G. Lambert, R. Fitour, V. Malka, A. Rousse, A. Beck, and E. Lefebvre, Femtosecond X-rays from laser-plasma accelerators, Rev. Mod. Phys. 85, 1 (2013).

[22] A. Pukhov and J. Meyer-ter-Vehn, Laser wake field acceleration: The highly non-linear broken-wave regime, Appl. Phys. B Lasers Opt. 74, 355 (2002).

[23] W. Lu, C. Huang, M. Zhou, W. B. Mori, and T. Katsouleas, Nonlinear Theory for Relativistic Plasma Wakefields in the Blowout Regime, Phys. Rev. Lett. 96, 165002 (2006).

[24] W. Lu, M. Tzoufras, C. Joshi, F. S. Tsung, W. B. Mori, J. Vieira, R. A. Fonseca, and L. O. Silva, Generating multi-GeV electron bunches using single stage laser wakefield acceleration in a
3D nonlinear regime, Phys. Rev. ST Accel. Beams 10, 061301 (2007).

[25] I. Kostyukov, E. Nerush, A. Pukhov, and V. Seredov, Electron Self-Injection in Multidimensional Relativistic-Plasma Wake Fields, Phys. Rev. Lett. 103, 175003 (2009).

[26] S. Kalmykov, S. A. Yi, V. Khudik, and G. Shvets, Electron SelfInjection and Trapping into an Evolving Plasma Bubble, Phys. Rev. Lett. 103, 135004 (2009).

[27] Y. Glinec, J. Faure, A. Lifschitz, J. M. Vieira, R. A. Fonseca, L. O. Silva, and V. Malka, On the properties of synchrotron-like Xray emission from laser wakefield accelerated electron beams, Europhys. Lett. 81, 64001 (2008).

[28] A. Popp, J. Vieira, J. Osterhoff, Z. Major, R. Hörlein, M. Fuchs, R. Weingartner, T. P. Rowlands-Rees, M. Marti, R. A. Fonseca, S. F. Martins, L. O. Silva, S. M. Hooker, F. Krausz, F. Gruner, and S. Karsch, All-Optical Steering of LaserWakefield-Accelerated Electron Beams, Phys. Rev. Lett. 105, 215001 (2010).

[29] C. Thaury, E. Guillaume, S. Corde, R. Lehe, M. Le Bouteiller, K. Ta Phuoc, X. Davoine, J. M. Rax, A. Rousse, and V. Malka, Angular-Momentum Evolution in Laser-Plasma Accelerators, Phys. Rev. Lett. 111, 135002 (2013).

[30] C. A. Peters, Statistics for Analysis of Experimental Data, Environmental Engineering Processes Laboratory Manual, AEESP, Champaign, IL (2001) https://www.princeton.edu/ cap/AEESP_Statchap_Peters.pdf.

[31] S. P. D. Mangles, A. G. R. Thomas, M. C. Kaluza, O. Lundh, F. Lindau, A. Persson, F. S. Tsung, Z. Najmudin, W. B. Mori, C.-G. Wahlström, and K. Krushelnick, Laser-Wakefield Acceleration of Monoenergetic Electron Beams in the First Plasma-Wave Period, Phys. Rev. Lett. 96, 215001 (2006).

[32] M.-H. Cho, Y.-K. Kim, H. Suk, B. Ersfeld, D. A. Jaroszynski, and M. S. Hur, Strong terahertz emission from electromagnetic diffusion near cutoff in plasma, New J. Phys. 17, 043045 (2015).

[33] A. Döpp, B. Mahieu, A. Lifschitz, C. Thaury, A. Doche, E. Guillaume, G. Grittani, O. Lundh, M. Hansson, J. Gautier, M. Kozlova, J. P. Goddet, P. Rousseau, A. Tafzi, V. Malka, A. Rousse, S. Corde, and K. T. Phuoc, Stable femtosecond X-rays with tunable polarization from a laser-driven accelerator, Light: Sci. Appl. 6, e17086 (2017). 